\title{
El Juego de rol "El puesto es tuyo" como herramienta para el desarrollo de competencias transversales y de acercamiento al ámbito profesional.
}

\author{
MariLuz Gordo ${ }^{a}$, Marta Fernández-Diego ${ }^{a}$, Leonor Ruiza, Llanos Cuenca ${ }^{a}$, Andrés \\ Boza $^{a}$, aDepartamento de Organización de Empresas. Universitat Politècnica de \\ València. Equipo de Innovación Educativa AEGECATE. (magormon@upvnet.upv.es \\ , marferdi@omp.upv.es,Iruiz@omp.upv.es,llcuenca@omp.upv.es, aboza@omp.upv ).
}

\begin{abstract}
The acquisition of competences is a recurrent issue in educational processes over the last few years. It becomes especially relevant with transversal competencies, as companies increasingly ask for the improvement of the socalled soft skills. Thus, they have been continuously promoted by universities. The aim of this paper is to propose an activity based on the role-playing technique which allows both, the internalization of concepts related to professionalism (which is taught in the Degree of Computer Engineering, in the framework of the subject "Deontology and Professionalism") and the development of the competences "Innovation, Creativity and Entrepreneurship" and "Teamwork and Leadership".

The activity focuses on the definition of a computer engineer's job, the elaboration of the curriculum vitae and the subsequent job interview to apply to that position. All this is carried out in a structured way, providing the students with entertainment and encouraging their initiative and reflection at the same time. Although it requires an important previous work on behalf of the teacher and an exhaustive monitoring and support in the classroom, the activity has been pleasantly welcomed by the students and the results obtained are satisfactory.
\end{abstract}

Keywords: role-playing, professionalism, competences, teamwork, innovation and creativity

\section{Resumen}

Dada la importancia de adquisición de competencias en el proceso educativo $y$ en especial la de las competencias transversales, ampliamente demandadas por el mundo profesional y promovidas desde el ámbito universitario, este trabajo propone la realización de una actividad basada en la metodología del juego de rol que permite de manera integrada la interiorización de conceptos relacionados con la materia de profesionalismo (impartida en el marco de la asignatura Deontología y Profesionalismo de segundo curso del 
El Juego de rol "El puesto es tuyo" como herramienta para el desarrollo de competencias transversales y de acercamiento al ámbito profesional

Grado de Ingeniería Informática) y el desarrollo de las competencias "Creatividad, Innovación y Emprendimiento innovación," y "Trabajo en Equipo y Liderazgo".

La actividad se centra en la definición de un puesto de trabajo de ingeniero informático, la elaboración de un currículum vitae para responder a este, y la preparación y posterior desarrollo de una entrevista de trabajo para optar al citado puesto. Todo ello se desarrolla de una manera pautada que a la vez permite el entretenimiento, la iniciativa y reflexión por parte del alumnado. Si bien requiere de un importante trabajo previo por parte del docente y un seguimiento y apoyo exhaustivo en el aula, la actuación ha sido gratamente aceptada por los estudiantes y los resultados obtenidos son satisfactorios.

Palabras clave: juego de rol, profesionalismo, competencias, trabajo en equipo, innovación y creatividad

\section{Introducción}

Resulta ineludible la búsqueda de soluciones que incrementen la participación del alumnado en aras de fomentar un aprendizaje significativo en el que la forma de trasladar conocimiento sea experiencial y por tanto los conceptos sean interiorizados de manera más profunda y por ende, eficaz. Guiados por esta visión los docentes investigamos nuevas maneras de hacer las cosas para conseguir distintos resultados, en aras de mejorar los actuales. La "gamificación" es una de las líneas por las que apostamos en la asignatura Deontología y Profesionalismo que impartimos en el $2^{\circ}$ curso del Grado de Ingeniería Informática de la ETSIINF en la Universitat Politècnica de València.

Con la implementación de la gestión por competencias en el mundo profesional y su traslado al universo docente se refuerza la necesidad de replantear el enfoque formativo indicado anteriormente, para que de una forma objetivable se consiga el desarrollo y la mejora continua de aptitudes por parte del colectivo estudiantil. Las competencias definidas como transversales por parte de la Universitat Politècnica de València requieren la potenciación de habilidades que necesariamente implican la puesta en marcha de actuaciones diversas y sobre todo la traslación a la práctica.

Acudiendo al Diccionario de la RAE (Real Academia Española), si bien no reconoce el térmico "Gamificación", proveniente del anglicismo "gamification", al ser partidaria la institución de expresar con palabras del idioma propio las nuevas realidades que van surgiendo en los distintos ámbitos (propone jueguización y juguetización o aprovechar combinaciones de vocablos ya existentes como mecánicas del juego), sí que dentro de las variantes del término juego, que define como "Acción y efecto de jugar como entretenimiento", reconoce como acepción la locución "juego de rol" delimitada como "juego en el que los participantes interpretan el papel de un personaje de ficción, en una historia de carácter misterioso o fantástico". 
Muchos autores han profundizado en el concepto y no son pocos los trabajos ya existentes que introducen la utilización de estas técnicas y la utilidad de trasladarlas al aula.

Como ya se planteó en un trabajo anterior de los autores "Simulación y Juegos de rol como herramienta de innovación en la asignatura Deontología y Profesionalismo" (Gordo et al., 2017), se considera interesante hacer mención del artículo de García et al. (2011) "la interdisciplinariedad en la educación superior: Propuesta de una guía para el diseño de juegos de rol" que introduce, tras hacer mención de algunas técnicas que favorecen el trabajo colaborativo, lo siguiente

Además de estas técnicas, se encuentra el uso de la simulación en el proceso de enseñanza aprendizaje, que, según Ruben (1999), basándose en los enfoques de aprendizaje de finales de los años 60, es una metodología que supera las limitaciones de la enseñanza tradicional. De hecho, y de acuerdo con este autor, el uso de las simulaciones como metodología educativa conlleva una mejora en el proceso de aprendizaje del propio estudiante, facilita la interactividad entre los estudiantes, el trabajo colaborativo y, en definitiva, un aprendizaje más activo.

Respecto a la competencia La innovación, creatividad y emprendimiento, retomamos asimismo la mención a los autores Jiménez y Moncholi (2009):

La sociedad ya ha tomado conciencia de que la creatividad es un derecho y una exigencia social; esta creatividad, indispensable en muchas disciplinas, puede desarrollarse a través de la técnica de juegos de rol, con la que, además, se potencia el aprendizaje y se suscita la curiosidad de los jugadores. Los juegos de rol, a pesar de que los personajes y la situación que se simula vienen definidos a priori, atendiendo a determinadas reglas impuestas por un director (normalmente el profesor o grupo de profesores que imparten una determinada materia), no exigen un diálogo concreto a los jugadores, siendo éstos libres para poder reconducir la situación o problemática planteada en aras de encontrar ciertas soluciones.

Aportaciones que contribuyen a confirmar que estas técnicas facilitan el desarrollo de las competencias "Creatividad, Innovación y Emprendimiento innovación," (CT4) y "Trabajo en Equipo y Liderazgo" (CT6).

En este caso la innovación docente que proponemos pretende aunar en una misma actividad el fomento de las citadas competencias competencia en el marco de la asignatura Deontología y Profesionalismo. Asignatura que se imparte en el segundo año del Grado de Ingeniería Informática de la Escuela Técnica Superior de Ingeniería Informática. La asignatura se divide en tres bloques: "Profesionalismo", "Aspectos legales" y "Ética". La actividad que se presenta está ligada al primer bloque, "Profesionalismo" que introduce el estudio de la "profesión en general" para posteriormente particularizar el análisis en el profesional informático. El tema repasa someramente la historia de la informática y analiza cuándo la informática comenzó a ser consciente de su existencia desvinculada de otras profesiones, presenta el mundo profesional para un profesional TI (Tecnologías de la 
El Juego de rol "El puesto es tuyo" como herramienta para el desarrollo de competencias transversales y de acercamiento al ámbito profesional

Información) y los distintos agentes y elementos participantes, para finalmente centrarse en estudiar las competencias en el ámbito de las TI.

\section{Objetivos}

El objetivo del presente trabajo es exponer el diseño y desarrollo de una actividad de trabajo en grupo y basada en simulación y juego de rol, llevada a cabo en la asignatura Deontología y Profesionalismo para la adquisición de diversas competencias por parte de los alumnos. Principalmente las competencias transversales creatividad, innovación y emprendimiento y la de trabajo en equipo. Asimismo la actividad es susceptible de trabajar otras competencias transversales, si bien, se ha considerado conveniente centrar el objetivo en las dos mencionadas.

\section{Desarrollo de la innovación}

Si bien el profesorado participante dispone de cierta experiencia en la aplicación de esta técnica, todavía sigue siendo una gran desconocida para los alumnos. Su aplicación al contexto de esta asignatura se considera de alto interés para conseguir la implicación del alumnado y con ello la interiorización de conceptos desde su puesta en práctica alejándonos de la pura transmisión teórica de contenidos.

En esta actividad en concreto, tratamos de llevar al aula una situación que con total seguridad va a ser vivida por los alumnos durante sus estudios si pretenden compatibilizar trabajo y carrera, y sin duda cuando estos se finalicen y opten a un puesto de trabajo en una empresa o entidad. Ponerlos en situación es totalmente novedoso para ellos. La preparación de la entrevista, la defensa del curricíulum, la participación en la propia entrevista y la reflexión posterior son fases que vivenciarán por primera vez (para la gran mayoría) durante la actividad, y gracias a ello es posible promover ciertas maneras de actuar que podrán serles de utilidad en su devenir profesional.

El proceso de adquisición de la competencia creatividad, innovación y emprendimiento se considera basado en las siguientes actuaciones:

1. Búsqueda de información sobre el contenido del puesto de trabajo facilitado por el docente.

2. Creación de una presentación de empresa y currículum en formato poco convencional.

3. Establecer pautas para la realización de la entrevista, idear una compilación de preguntas clave para identificar la valía del candidato y su adecuación al puesto.

4. Originalidad en la presentación del candidato. Los alumnos que jueguen el rol del candidato deberán ponerse en situación de intentar conseguir el empleo y para ello deben preparar una exposición adecuada, destacando aquello más valorable y manifestando su capacidad e interés para y por el puesto 
En cuanto a la competencia trabajo en equipo, los alumnos trabajan durante la totalidad de la actividad de forma cooperativa bajo el rol que les ha sido asignado o que el profesor les ha permitido libremente repartirse. Compaginan, en esta tarea la colaboración en el desarrollo de un documento (asociado a la Fase I que se explica más adelante), con la participación con el compañero en la entrevista (debiendo respetar cada uno los tiempos del otro, así como la continuidad en el argumentario) y por último con el establecimiento de consenso en la redacción de las conclusiones.

La actividad ha sido creada íntegramente por los profesores basándose en la experiencia en la docencia de la asignatura.

\subsection{Estructura de la actividad}

Se organiza a los alumnos en grupos de cuatro personas. Si bien en las diferentes ocasiones que se ha planteado la actividad se ha permitido que los alumnos se organicen de forma autónoma con cierta participación del profesor para uniformizar los grupos, se puede plantear la organización directa de los grupos por parte del profesor.

Se les explica la actividad que tiene por título "El puesto es tuyo". Asimismo se les entrega un documento con la explicación de la actividad y un pequeño papel indicando el puesto de trabajo sobre el que deben trabajar.

Cada grupo de cuatro alumnos se dividirán en dos grupos y por pareja asumirán uno de estos dos roles: Rol responsable RRHH de una empresa y Rol candidato.

El trabajo costa de diferentes fases:

Fase 1: Preparación presentación empresa y currículum del candidato. 30 minutos

Trabajarán por separado cada uno de los roles y desarrollarán en paralelo lo siguiente:

- Los responsables de RRHH elaborarán una presentación para recabar candidatos, donde incluirán información de la empresa y del puesto que pretenden cubrir. Deberán concebir desde el nombre de la empresa a la totalidad de la descripción del puesto, si bien disponen de acceso a internet para facilitarles la generación de ideas. Los términos en que se les plantea la tarea son: 
El Juego de rol "El puesto es tuyo" como herramienta para el desarrollo de competencias transversales y de acercamiento al ámbito profesional

Sois una empresa en búsqueda de informáticos. Tenéis un nuevo proyecto y necesitáis los mejores profesionales. Debéis de ser conscientes de que el profesional informático está altamente demandado. Desde que se impuso el grado los estudiantes se colocan como churros y hay que saber "vender" el proyecto con el fin de cubrir los puestos con esta titulación.

Tenéis previsto una exposición en la escuela de informática y tenéis que elaborar una presentación. Habéis pensado que el ppt está muy visto y para que sea rompedora e impactante emplearéis Prezi $\mathrm{u}$ otro formato innovador. Ni que decir que la presentación debe ser atractiva para captar a los candidatos y lógicamente a los mejores profesionales.

Los puestos a cubrir son:

Deberéis indicar:

- Nombre del puesto a cubrir (os lo indicará el/la profesor/a)

- Funciones

- Competencias, divididas en:

- Conocimientos

- Experiencia

- Habilidad

- Identificar si se requieren -Hard Skills o Soft Skills

- Salario

Figura 1 Guión para alumnos rol "responsables RRHH"

- Los candidatos elaboraran su currículum personal para optar al puesto. Todos los datos que elaboren serán inventados, desde el nombre del candidato a cualquier otro contenido del currículum, si bien pueden basarse en información existente. El documento guión que se les facilita es el siguiente.

Sois un titulado en informática y andáis intentando cambiar de puesto de trabajo. Sois bueno, uno de los mejores, y no solo lo dices tú, lo dice tú abuela. Tenéis amplia experiencia en diversos campos y mucho empuje para acometer nuevos proyectos, por tanto estáis seguros de que las empresas quedarán seducidas por vuestro historial y vuestra ilimitada ristra de competencias. No obstante sois conscientes de que hay mucho friki suelto y tenéis que atar bien la puesta en escena y la entrevista, no vais a mandar el típico currículum soporífero, sino que habéis pensado dar la campanada montando vuestro cv en prezi o similar destacando lo que os hace mejores para el puesto.

Deberéis indicar:

- Nombre del puesto al que se opta (os lo indicará el/la profesor/a)

- Funciones

- Competencias, divididas en:
- Conocimientos
- Experiencia
- Habilidad

- Si se dispone de hard Skills o soft Skills

- Salario deseable

Figura 2 Guión para alumnos rol "candidato"

(c) BY-NC-ND 2019, Universitat Politècnica de València 
Como se puede observar se hace especial hincapié en la definición de las competencias para el puesto, así como en la diferenciación de las Hard y Soft skills (Hard competencias más vinculadas a lo conceptual y las Soft, asociadas a las habilidades, y por tanto en línea con las competencias transversales)

Ambos trabajarán sobre el puesto indicado en el papel que entrega el docente. Aquí se fomenta que los alumnos investiguen sobre puestos de trabajo vinculados con la gestión, que son menos familiares para ellos como informáticos porque se alejan del formato de diseñador, analista, programador... y que sin embargo tienen una demanda importante en el mundo empresarial, como por ejemplo: consultor SAP, auditor norma ISO 27001, asesor en protección de datos, etc.

Asimismo, se fomenta la creatividad intentando que sean imaginativos en los formatos de presentación y en la forma de presentar los contenidos. Nuevas formas de presentaciones alejadas del formato power point, como por ejemplo Prezi pueden ser investigadas y empleadas. Puesto que son estudiantes de informática y viven inmersos en el mundo del software agradecen trabajar con nuevas herramientas y tienden a familiarizarse rápidamente con ellas.

Fase II Preparación de la entrevista 15 minutos.

El docente ofrecerá unas pautas básicas sobre el objetivo y la forma en que debería desarrollarse una entrevista de trabajo: trato por ambas partes desde el respecto, profesionalidad por parte de los responsables de RRHH, tranquilidad por parte del candidato y por supuesto preparación previa para asegurar el mejor desempeño de la misma.

En esta fase juega un importante papel el aspecto creativo, ya que cada rol deberá elaborar una guía para el desarrollo de la entrevista. El rol Responsables RRHH explicará la presentación, permitirá que el candidato se presente posteriormente y procederá a consultar aquellas cuestiones que considere de interés para decidir sobre la adecuación al puesto.

Fase III: Desarrollo de la entrevista: 20 minutos

Con la puesta en práctica de actividades similares se ha llegado a la conclusión que hay dos aspectos claves para asegurar la adopción del rol asignado: Definir claramente el momento en que se debe tomar conciencia del rol y disponer de un espacio acorde a la situación que se les pide desarrollar. Para ello se apuntan las siguientes pautas de actuación:

1) A los alumnos que actúan como candidatos se les invita a salir del aula y a esperar fuera hasta que uno de los responsables de RRHH acuda a recibirlos.

2) Se reubicará el mobiliario con el fin de permitir el formato entrevista lo más real posible, es decir, cara a cara, frente a frente, y sin obstáculos visuales, entrevistadores y candidatos. Asimismo se debe asegurar que ambos roles tengan acceso a la presentación y currículum para emplearlo y consultarlo en la entrevista, respectivamente. 
El Juego de rol "El puesto es tuyo" como herramienta para el desarrollo de competencias transversales y de acercamiento al ámbito profesional

3) Una vez esté organizado, el profesor indicará a los responsables que tienen un candidato en recepción que indica que se le ha citado para una entrevista. Uno de los representantes de RRHH deberá acudir a recibirlos,

4) Una vez consideren los alumnos que se ha completado la entrevista (normalmente decisión de los estudiantes que ejerzan el rol de responsables de RRHH) uno de los responsables de la empresa acompañará a los alumnos a la puerta (recepción). Y entonces todos volverán a ocupar el rol de alumno.

Con esto se consigue que quede claramente identificado el momento en que se da el cambio de rol y la toma de conciencia del mismo, un aspecto que aunque parezca poco importante resulta imprescindible para asegurar el correcto desarrollo de la actividad, más si los grupos los han elegido ellos y son propicios al regocijo. Se trata de definir claramente el INICIO y FIN de la asunción de rol.

Se apunta asimismo, como recomendación, que en caso de que algún grupo de alumnos no esté desarrollando adecuadamente la entrevista (normalmente porque les resulta graciosa la situación y les invade la risa), el docente intervenga de manera comprensiva, pidiendo que vuelvan a salir de aula e intenten tomar de nuevo conciencia del cambio de rol, animándoles a controlarse, para poder realizar la entrevista de acuerdo a lo esperado y planificado. Es imprescindible en esta fase el desarrollo de la competencia Trabajo en equipo, permitiendo dentro de cada rol la participación del compañero, reforzando sus ideas, manifestando las propias y llegando a un consenso.

Fase IV: Reflexión y Evaluación por parte de los alumnos. 15 minutos

La última fase consiste en reflexionar en base a un formato facilitado, sobre el desarrollo de la entrevista. El formato se plantea de la siguiente forma:

Para el alumnado que ha ejercido como Candidato: 


\begin{tabular}{|l|l|l|l|l|l|}
\hline $\begin{array}{l}\text { Equipo CANDIDATO } \\
\text { valora los siguientes conceptos } \\
\text { del equipo EMPRESA, } \\
\text { para el puesto: }\end{array}$ & \multicolumn{4}{l|}{ Alumnos del equipo CANDIDATO } \\
\hline $\begin{array}{l}\text { 1=Puntuación mínima } \\
\text { 5=Puntuación máxima }\end{array}$ & 1 & 2 & 3 & 4 & 5 \\
\hline $\begin{array}{l}\text { Calidad del documento } \\
\text { presentación }\end{array}$ & & & & & \\
\hline $\begin{array}{l}\text { ¿Es creativo? } \\
\text { Claridad en la exposición }\end{array}$ & & & & & \\
\hline Habilidad para vender el puesto & & & & & \\
\hline $\begin{array}{l}\text { ¿De verdad queremos trabajar } \\
\text { para ellos? } \\
\text { ¿Por qué? }\end{array}$ & I & & & & \\
\hline Algo a destacar & & & & \\
\hline
\end{tabular}

Figura 3 Plantilla evaluación alumnos rol candidato

Para el alumnado que ha ejercido como Responsables de RRHH:

\begin{tabular}{|c|c|c|c|c|c|}
\hline $\begin{array}{l}\text { Equipo EMPRESA } \\
\text { valora los siguientes } \\
\text { conceptos del equipo } \\
\text { CANDIDATO, } \\
\text { para el puesto: }\end{array}$ & $\begin{array}{l}\text { Alumn } \\
- \\
-\end{array}$ & qu & PRE & & \\
\hline $\begin{array}{l}\text { 1=Puntuación mínima } \\
\text { 5=Puntuación máxima }\end{array}$ & 1 & 2 & 3 & 4 & 5 \\
\hline $\begin{array}{l}\text { Calidad del documento } \\
\text { presentación }\end{array}$ & & & & & \\
\hline ¿Es creativo? & & & & & \\
\hline Claridad en la exposición & & & & & \\
\hline Habilidad para venderse & & & & & \\
\hline $\begin{array}{l}\text { ¿Lo contratamos? } \\
\text { ¿Por qué? }\end{array}$ & & & & & \\
\hline Algo a destacar & & & & & \\
\hline
\end{tabular}

Figura 4 Plantilla evaluación alumnos rol empresa

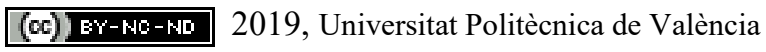


El Juego de rol "El puesto es tuyo" como herramienta para el desarrollo de competencias transversales y de acercamiento al ámbito profesional

Asimismo aquí se refuerza la competencia trabajo en equipo al tener que consensuar con el compañero que se desarrolla el rol las respuestas y la toma de decisión y su argumentación.

Evaluación de la actividad:

Los alumnos deberán remitir al docente los siguientes documentos: Presentación RRHH y currículum, así como las dos encuestas, una correspondiente a cada uno de los roles que conforman el equipo.

La evaluación planteada es la siguiente:

-Documentación correspondiente a la Fase I Presentación empresa y curriculum vitae $40 \%$

-Evaluación por parte de cada rol del rol contrario en la entrevista: $40 \%$

-Observación del profesor durante el desarrollo de la entrevista: $20 \%$

Se ha consultado a los alumnos sobre el desarrollo de la actividad, para ello se ha introducido un sencillo cuestionario al final de la actividad y las respuestas han sido analizadas.

\begin{tabular}{|l|c|c|c|}
\hline \multicolumn{3}{|c|}{ AYUDANOS A MEJORAR EVALUANDO LA PRÁCTICA } \\
\hline La práctica ha sido & $(-)$ & $(-)$ & ( ) \\
\hline Amena & & & \\
\hline Interesante & & & \\
\hline $\begin{array}{l}\text { Ayuda a asentar la } \\
\text { teoría }\end{array}$ & & & \\
\hline $\begin{array}{l}\text { ¿Puedes mejorarla? } \\
\text { ¿Qué cambiarías? }\end{array}$ & & & \\
\hline
\end{tabular}

GRACIAS!!

Figura 5 Plantilla cuestionario actividad

\section{Resultados}

\subsection{Contexto y desarrollo}

En la asignatura cuatrimestral de segundo curso del Grado de Ingeniería Informática de la Escuela Técnica Superior de Ingeniería Informática, Deontología y Profesionalismo, se aplicó la actividad "El Puesto es Tuyo".

La actividad propuesta pretendía que los alumnos fueran capaces preparar una demanda de puesto de trabajo y un currículum vitae y defenderlos a continuación en el desarrollo de una 
entrevista de trabajo. El profesor facilita diferentes documentos, así como pautas orales y seguimiento exhaustivo de cada grupo, con el fin de guiar la implementación de la metodología por parte de los estudiantes.

Las competencias "innovación, creatividad y emprendimiento" (CT4) y "Trabajo en Equipo y Liderazgo" (CT6) son competencias absolutamente necesarias en el terreno profesional en el marco de la ingeniería y por tanto resulta de especial relevancia su fomento en el periodo universitario. Consideramos que actividades como la presente contribuyen a ello.

\subsection{Resultados de la innovación}

\section{Evaluación}

Los alumnos han obtenido buenos resultados en las calificaciones de la actividad, ya que las presentaciones han sido originales en general. Han fallado en olvidarse en ocasiones la diferenciación entre Hard o Soft skills. Respecto al desarrollo de la entrevista se ha conseguido la implicación de los mismos en el rol representado y todos los grupos han llevado a cabo la entrevista de acuerdo con el guión planteado.

La actitud general del alumnado es muy proactiva y el ambiente general satisfactorio, si bien durante el desarrollo de la entrevista se genera cierto caos y subida de tono que tiene que ser manejada hábilmente por el docente.

A partir del análisis de las encuestas realizadas al alumnado se pueden valorar aspectos de interés. Los alumnos han sido críticos con la utilidad de la actividad para asentar los conocimientos teóricos facilitados en la exposición magistral previa y han opinado que el factor tiempo facilitado para cada una de las fases es muy mejorable, pero sin embargo una amplia cantidad de alumnos ha calificado la actividad ha sido amena e interesante e incluso han aportado mejoras como: facilitar la actividad con tiempo, establecer un sistema de puntuación por parte de la empresa en relación al nivel de cumplimiento de las competencias, etc. Medidas que serán estudiadas para incorporarlas, en la medida de lo posible, en próximas ediciones de la actividad.

\section{Evidencias}

Se muestran a continuación algunas de las evidencias recogidas durante el desarrollo de la actividad. 
El Juego de rol "El puesto es tuyo" como herramienta para el desarrollo de competencias transversales y de acercamiento al ámbito profesional

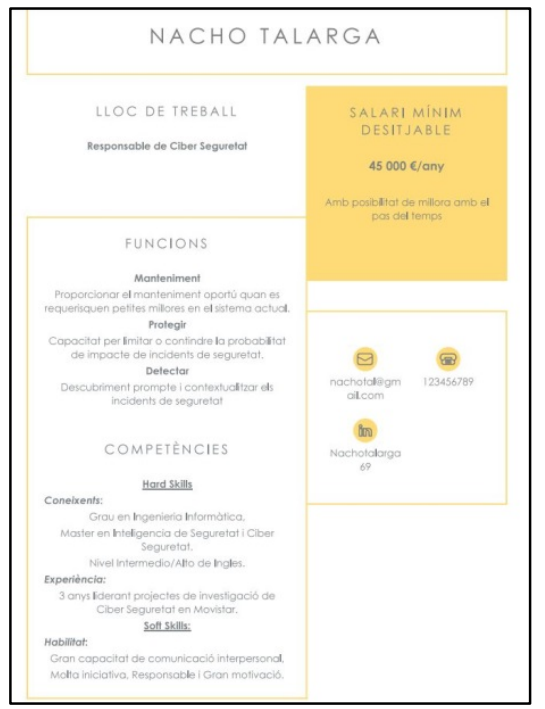

Figura 6 Ejemplo evidencia currículum vitae

\begin{tabular}{|c|c|c|c|c|c|}
\hline L'EMPRESA & \multirow{2}{*}{\multicolumn{5}{|c|}{ 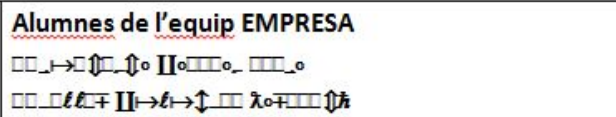 }} \\
\hline CalaNejos S. A. & & & & & \\
\hline $\begin{array}{l}\text { valora els següents } \\
\text { conceptes del CANDIDAT }\end{array}$ & \multirow{2}{*}{\multicolumn{5}{|c|}{ 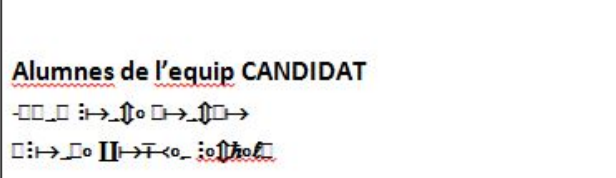 }} \\
\hline Consultor SAP & & & & & \\
\hline $\begin{array}{l}\text { 1=Puntuació mínima } \\
\text { 5=Puntuació màxima }\end{array}$ & 1 & 2 & 3 & 4 & 5 \\
\hline $\begin{array}{l}\text { Qualitat del document } \\
\text { presentació }\end{array}$ & & & & & $\mathrm{x}$ \\
\hline És creatiu? & & & & & $\mathrm{x}$ \\
\hline Claredat en l'exposició & & & & & $\mathrm{x}$ \\
\hline Habilitat per a vendre's & & & & & $\mathrm{x}$ \\
\hline $\begin{array}{l}\text { Ho contractem? } \\
\text { Per què? }\end{array}$ & \multicolumn{5}{|c|}{$\begin{array}{l}\text { Si, han sabut transmitir les seues capacitats per al càrrec amb } \\
\text { seguretat. A més, tenen una extensa experiència laboral } \\
\text { treballant en grans empreses i vivències a l'estranger. }\end{array}$} \\
\hline Alguna cosa a destacar & \multicolumn{5}{|c|}{$\begin{array}{l}\text { Els han recomanat en varies ocasions com a excelents } \\
\text { directius i han cumplit perfectament les nostres expectatives. }\end{array}$} \\
\hline
\end{tabular}

Figura 7 Ejemplos evidencias cuestionario evaluación alumnos rol empresa.

(c)) EY-NC-ND 2019, Universitat Politècnica de València 


\section{Limitaciones}

- La falta de experiencia en la puesta en práctica de esta técnica podría ser una de las limitaciones, ya que de entrada genera, en ocasiones, falta de predisposición por cierta parte del alumnado hacia la adopción de nuevos roles debido a la carencia de la vertiente teatral en los alumnos. Esta cuestión merece refuerzo positivo por parte del docente y se supera conforme se avanza en el desarrollo de la actividad.

- La realización en grupos grandes genera bastantes altercados debido al movimiento y ajetreo, así como dificulta la actuación de guía y testigo del profesor

\section{- 5. Conclusiones}

La actividad "EL PUESTO ES TUYO" que se ha realizado en la asignatura cuatrimestral de segundo curso del Grado de Ingeniería Informática de la ETSIINF, Deontología y Profesionalismo ha contribuido a que el estudiante tenga un mejor conocimiento de su futuro contexto profesional mediante la aplicaron las técnicas de juegos de rol. La actividad se evalúa dentro de las tareas a realizar por el alumnado, vinculadas a la parte teórica de la asignatura.

La utilización de la técnica ha permitido de forma directa el desarrollo de las competencias "innovación, creatividad y emprendimiento" (CT4) y "Trabajo en Equipo y Liderazgo" (CT6) en la preparación y desarrollo de una entrevista de trabajo por parte de los estudiantes, para lo que se ha pretendido simular una situación lo más ajustada a la realidad en la que ellos son los actores principales.

No debe olvidarse que el planteamiento de este tipo de actividad requiere de un esfuerzo importante por parte del docente debido a la preparación y al seguimiento constante a los alumnos para guiarles y realizar la evaluación de la forma más objetiva posible. Frente a una actividad que simplemente les requiera realizar un determinado trabajo y se realice la habitual corrección y evaluación posterior, la inversión es notable en términos de tiempo de dedicación, si bien la implicación de los alumnos y clima de entretenimiento-aprendizaje que se respira en el aula es un aliciente innegable, y por tanto la energía invertida es gratamente recompensada en la mayor parte de los casos. Eso sin mencionar el reto que supone para el docente el descubrir nuevas maneras de hacer las cosas, la oportunidad de poderlas llevar a la práctica y observar como los alumnos se sienten estimulados con su desarrollo y a la vez son capaces de valorar de forma crítica la actividad presentada y ayudarnos a completarla y mejorarla.

Los resultados obtenidos se consideran resultan satisfactorios, tanto en cuanto a calificaciones como a valoración por parte de los alumnos. Los alumnos en general han elaborado de forma creativa y correcta la documentación solicitada y han evaluado positivamente la participación del candidato y el planteamiento de la entrevista por parte de los responsables de RRHH, respectivamente. El docente ha valorado satisfactoriamente la parte correspondiente a la intervención en el aula. La mayor parte de los alumnos han obtenido la máxima calificación en la actividad. 
El Juego de rol "El puesto es tuyo" como herramienta para el desarrollo de competencias transversales y de acercamiento al ámbito profesional

Asimismo, como aspectos a considerar en futuras aplicaciones, cabe depurar más la técnica y la actividad con el fin de ajustarla principalmente a tiempos más cómodos para los participantes, así como replantearse la organización de la propia sala para facilitar la ubicación de los personajes de la manera más adecuada así como la disposición de los soportes necesarios durante la ejecución de la entrevista (guion responsables RRHH y currículum candidato)

\section{- 6. Referencias}

Game Marketing <http://www.gamkt.com/2011/12/09/gamificacion-segun-la-rae/> [Consulta: 23 de marzo de 2019].

García Magna, D., Castillo Rodríguez, C., Rios Moyano, S., Cristofol Rodríguez, C., Carrasco Santos, M., Rodríguez Mérida, R. M., ... \& Pastor García, I. (2011). La interdisciplinariedad en la educación superior: propuesta de una guía para el diseño de juegos de rol. Teoría de la Educación,12(1)27.

Gordo M., Fernadez-Diego M, Alarcón F., Boza A. (2017). “ Simulación y Juegos de rol como herramienta de innovación en la asignatura Deontología y Profesionalismo". En : In-Red 2017. III Congreso Nacional de innovación educativa y de docencia en red. (Julio 13-14, 2017. Valencia) Riunet. Universitat Politècnica de València https://riunet.upv.es/handle/10251/106102

Jiménez Martín, S. y Moncholi Chaparro, M.A. (2009). El entrenamiento en técnicas creativas en el Espacio Europeo de Educación Superior. Actas del I Congreso Internacional Latina de Comunicación Social (pp. 1-12) Universidad de La Laguna. http://www.revistalatinacs.org/09/Sociedad/actas/12silvia.pdf

Real Academia Española < http://www.rae.es/> [Consulta: 23 de marzo de 2019].

Ruben, B.D. (1999). Simulations, Games, and Experience Based Learning: The Quest for a New Paradigm for Teaching and Learning. Simulation and Gaming. 30 (4), 498505 . 